\title{
Knockdown of long non-coding RNA metastasis associated lung adenocarcinoma transcript 1 inhibits the proliferation and migration of bladder cancer cells by modulating the microRNA-34a/cyclin D1 axis
}

\author{
YE LIU $^{1 *}$, SAIYUE GAO ${ }^{2 *}$, QINGYAN DU ${ }^{2}$ and QINGSONG ZHAO ${ }^{3}$ \\ ${ }^{1}$ Clinical Laboratory, Jining No.1 People's Hospital, Jining, Shandong 272011; ${ }^{2}$ Physical Examination Laboratory, \\ Linyi Lanshan District Center for Disease Control and Prevention, Linyi, Shandong 276000; ${ }^{3}$ Department of Urology, \\ Jining No.1 People's Hospital, Jining, Shandong 272011, P.R. China
}

Received July 7, 2018; Accepted October 16, 2018

DOI: 10.3892/ijmm.2018.3959

\begin{abstract}
Long non-coding RNA (lncRNA) metastasis associated lung adenocarcinoma transcript 1 (MALAT1) has been demonstrated to participate in the development and progression of some common cancer types, including bladder cancer (BC). However, the regulatory mechanism of MALAT1 underlying BC growth and metastasis remains to be fully elucidated. The present study revealed that MALAT1 was significantly upregulated in BC tissues and cell lines compared with the adjacent non-tumour tissues and the normal urinary tract epithelial cell line SV-HUC-1, respectively. The expression levels of MALAT1 were higher in stage III-IV BC tissues when compared with that in stage I-II tissues. Furthermore, knockdown of MALAT1 significantly inhibited $\mathrm{BC}$ cell proliferation and migration by targeting microRNA (miR)-34a. The expression levels of miR-34a were significantly decreased in $\mathrm{BC}$ tissues and cell lines compared with that of adjacent non-tumour tissues and SV-HUC-1 cells. In addition, the expression of miR-34a was inversely correlated with the expression of MALAT1 in BC tissues. The present study revealed that cyclin D1 (CCND1) was identified as a target gene of miR-34a, and its expression was negatively mediated by miR-34a in BC cells. Notably, the upregulation of CCND1 impaired the effect of MALAT1 inhibition on BC cell proliferation and migration. In addition, the expression levels of CCND1 were significantly increased in BC tissues and cell
\end{abstract}

Correspondence to: Dr Qingsong Zhao, Department of Urology, Jining No.1 People's Hospital, 6 Jiankang Road, Jining, Shandong 272011, P.R. China

E-mail: sdzhaoqingsong@sina.com

*Contributed equally

Key words: bladder cancer, microRNAs, long non-coding RNAs, cyclin D1 lines. In conclusion, the present findings demonstrated that the knockdown of 1ncRNA MALAT1 inhibits the proliferation and migration of $\mathrm{BC}$ cells by modulating the miR-34a/CCND1 axis, suggesting that the MALAT1/miR-34a/CCND1 axis may be a potential therapeutic target for $\mathrm{BC}$ treatment.

\section{Introduction}

Bladder cancer (BC) is one of the most common human cancer types worldwide that results in a large number of cancer-associated fatalities each year $(1,2)$. Rapid tumour growth and metastasis are the primary reasons for the high mortality associated with patients with $\mathrm{BC}(2,3)$. Therefore, exploring the regulatory mechanisms underlying the proliferation and migration of BC cells may aid the development of novel therapeutic strategies for this disease $(2,3)$.

Long non-coding RNAs (lncRNAs) are a class of small non-coding RNAs that consist of $>200$ nucleotides (4). By regulating downstream effectors, including microRNAs (miRs) and proteins, lncRNAs serve important roles in a variety of biological processes, such as cell proliferation, survival, apoptosis, energy metabolism, migration, invasion and tumourigenesis (5-7). In the recent decade, a large number of lncRNAs have been reported to function as oncogenes or tumour suppressors in various human cancer types, including BC (8-10). LncRNA metastasis associated lung adenocarcinoma transcript 1 (MALAT1) is frequently deregulated in some common cancer types and has been revealed to serve as a tumour promotor or suppressor (11-13). For instance, MALAT1 is upregulated in osteosarcoma, and the knockdown of MALAT1 inhibits osteosarcoma cell proliferation and migration and induces cell apoptosis (11). In addition, MALAT1 has been reported to promote renal cell carcinoma cell proliferation and invasion, as well as inhibit cell apoptosis by modulating the miR-205/enhancer of zeste homolog 2 (Ezh2) axis (14). Furthermore, a previous study reported that MALAT1 promotes BC cell migration through the induction of epithelial-to-mesenchymal transition (EMT) (15). In addition, Li et al (16) identified that the high expression of MALAT1 
was associated with a poor prognosis and promoted clinical progression and metastasis in BC. Xie et al (17) demonstrated that MALAT1 promoted BC cell invasion and inhibited cell apoptosis via antagonizing miR-125b. However, it remains unclear whether other miRs and downstream proteins are also involved in MALAT1-mediated BC.

miRs are a type of non-coding small RNA that are 22-25 nucleotides in length. These RNAs directly bind to complementary sites in the 3'-untranslated region (UTR) region of target mRNAs and thus cause translation inhibition $(18,19)$. By affecting the expression of their target genes, miRs are also involved in various biological processes $(20,21)$. It is well-established that miR-34a generally functions as a tumour suppressor in human cancer (22). For instance, miR-34a inhibits the proliferation and promotes the apoptosis of non-small cell lung cancer (NSCLC) cells by targeting transforming growth factor $\beta$ receptor 2 (TGF- $\beta$ R2) (22). In addition, miR-34a inhibits osteosarcoma cell proliferation by the inhibition of ether à go-go 1 expression (23). In BC, Yu et al (24) demonstrated that miR-34a inhibited metastasis and angiogenesis by directly targeting cluster of differentiation 44. Sun et al (25) reported that miR-34a inhibited $\mathrm{BC}$ cell proliferation and invasion by targeting the orphan nuclear receptor hepatocyte nuclear factor $4 \gamma$. However, to the best of our knowledge the association between MALAT1 and miR-34a in BC has not been previously reported.

The present study aimed to investigate the clinical significance of MALAT1 expression in BC. In addition, the regulatory role of MALAT1 in BC growth and metastasis in vitro and the underlying mechanism were studied.

\section{Materials and methods}

Clinical tissues. The present study was approved by the Ethics Committee of First People's Hospital of Jining City (Jining, China). BC and matched adjacent non-tumor tissues were collected from a total of 56 patients with primary BC at the First People's Hospital of Jining City between April 2011 and March 2013. Written informed consent was collected from all patients. These patients with $\mathrm{BC}$ included 34 male and 22 female, and were between 42 to 78 years old. All tissues were pathologically confirmed by the Department of Pathology at our hospital. No patients received chemotherapy and radiotherapy prior to surgical resection. Following surgical resection, the tissues were immediately frozen and stored at $-80^{\circ} \mathrm{C}$ until use.

Cell culture and transfection. BC cell lines (HT-1376, RT112, 253J and T24) and the normal urinary tract epithelial cell line SV-HUC-1 were purchased from the Chinese Academy of Sciences Cell Bank (Shanghai, China). Cells were cultured in Dulbecco's Modified Eagle's Medium (DMEM, Thermo Fisher Scientific, Inc., Waltham, MA, USA) with $10 \%$ fetal bovine serum (Thermo Fisher Scientific, Inc.) at $37^{\circ} \mathrm{C}$ in an atmosphere containing $5 \% \mathrm{CO}_{2}$.

For cell transfection, T24 cells were transfected with $100 \mathrm{nM}$ of negative control (NC) small interfering (si) RNA (siNC group; forward sequence, 5'-UUCUCCGAA CGUGUCACGUTT-3'; and reverse sequence, 5'-ACGUGA CACGUUCGGAGAATT-3'), 100 nM of MALAT1 siRNA
(siMALAT1 group; forward sequence, 5'-GCAAAUGAA AGCUACCAAU-3'; and reverse sequence, 5'-AUUGGU AGCUUUCAUUUGCTT-3'), $1 \mu \mathrm{g}$ of pcDNA3.1 vector (Amspring, Changsha, China), $100 \mathrm{nM}$ of miR-NC mimics (miR-NC group; cat. no. HMC0002, Sigma-Aldrich; Merck KGaA, Darmstadt, Germany), $100 \mathrm{nM}$ of miR-34a mimics (miR-34a group; cat. no. HMI0509, Sigma-Aldrich; Merck $\mathrm{KGaA}$ ), or co-transfected with siMALAT1 and NC inhibitor (siMALAT1+anti-NC group), siMALAT1 and miR-34a inhibitor (siMALAT1+anti-miR-34a group), siMALAT1 and $1 \mu \mathrm{g}$ of blank vector (siMALAT1+blank group; Amspring), siMALAT1 and CCND1 plasmid (siMALAT1+CCND1; Amspring), miR-NC and blank vector (miR-NC+blank group), miR-34a mimics and blank vector (miR-34a+blank group), and miR-34a mimics and CCND1 plasmid (miR-34a+CCND1 group) using Lipofectamine 2000 (Thermo Fisher Scientific, Inc.). At $48 \mathrm{~h}$ post-transfection, the cells were harvested and reverse transcription-quantitative polymerase chain reaction (RT-qPCR) was conducted to determine the transfection efficiency.

$R T-q P C R$. Total RNA was isolated from the tissues and cell lines using TRIzol reagent (Thermo Fisher Scientific, Inc.) according to the manufacturer's protocol. The total RNA $(1 \mu \mathrm{g})$ was reverse transcribed into cDNA using the PrimeScript RT Reagent Kit (Takara Bio, Inc., Otsu, Japan) according to the manufacturer's protocol. The miR-specific TaqMan miR assay kit (Applied Biosystems; Thermo Fisher Scientific, Inc.) was used to determine miR expression using the ABI 7500 Sequence Detection System (Thermo Fisher Scientific, Inc.). U6 was used as the internal control. For lncRNA and mRNA expression detection, PCR was performed using the SYBR-Green qPCR mix (Toyobo Life Science, Osaka, Japan) according to the manufacturer's protocol. Glyceraldehyde-3-phosphate dehydrogenase (GAPDH) was used as the internal control. The PCR reaction condition was as follows: $95^{\circ} \mathrm{C}$ for $5 \mathrm{~min}$ followed by 40 cycles of $95^{\circ} \mathrm{C}$ for $30 \mathrm{sec}$ and $60^{\circ} \mathrm{C}$ for $30 \mathrm{sec}$. The relative expression levels were calculated using the $2^{-\Delta \Delta \mathrm{Cq}}$ method (26). The primer sequences used were as follows: U6 forward, 5'-CTCGCTTCGGCAGCACATATACT-3' and reverse, 5'-CGCTTCACGAATTTGCGTGT-3'; MALAT1 forward, 5'-GGTAACGATGGTGTCGAGGTC-3' and reverse, 5'-CCAGCATTACAGTTC TTGAACATG-3'; CCND1 forward, 5'-GCTGCGAAGTGGAAACCATC-3' and reverse, 5'-CCTCCTTCTGCACACATTTGAA-3'; and GAPDH forward, 5'-GGAGCGAGATCCCTCCAAAAT-3' and reverse, 5'-GGCTGTTGTCATACTTCTCATGG-3'. The primers for miR-34a were purchased from Guangzhou Fulengen Co., Ltd. (cat. no. HmiRQP0439; Guangzhou, China) and the sequences were not supplied by the manufacturer.

Western blot analysis. Tissues and cells were lysed in cold radioimmunoprecipitation assay buffer (Thermo Fisher Scientific, Inc.). Protein concentration was examined using a BCA protein assay kit (Beyotime Institute of Biotechnology, Haimen, China). Proteins (50 $\mu \mathrm{g}$ per lane) were loaded onto $10 \%$ SDS-polyacrylamide gels and were blotted onto polyvinylidene difluoride membranes (Thermo Fisher Scientific, Inc.). Membranes were blocked with $5 \%$ non-fat dry milk in Tris-buffered saline with Tween-20 at room temperature 
for $2 \mathrm{~h}$. Following this, membranes were incubated at $4{ }^{\circ} \mathrm{C}$ overnight with rabbit monoclonal anti-CCND1 primary antibody (dilution 1:50; cat. no. ab134175; Abcam, Cambridge, MA, USA) and rabbit polyclonal anti-GAPDH primary antibody (dilution 1:100; cat. no. ab9485; Abcam). Following 3 wash steps with phosphate buffered saline with Tween-20, the membranes were incubated with horseradish peroxidase-conjugated goat anti-rabbit secondary antibody (dilution 1:5,000; cat. no. ab6721, Abcam) at room temperature for $1 \mathrm{~h}$. Subsequently, the proteins were visualized using an enhanced chemiluminescent kit (ECL-plus, Thermo Fisher Scientific, Inc.) and analysed using Image-Pro Plus software 6.0 (Media Cybernetics, Inc., Rockville, MD, USA).

Cell Counting Kit-8 (CCK-8) assay. A CCK-8 assay was conducted to study cell proliferation. Following $48 \mathrm{~h}$ of transfection, the cells $(5,000$ cells per well) were seeded onto 96 -well plates and cell proliferation was measured at $0,24,48$ and $72 \mathrm{~h}$ using CCK-8 (Thermo Fisher Scientific, Inc.) according to the manufacturer's instructions. The absorbance was detected at an optical density of $450 \mathrm{~nm}$ using a spectrophotometer.

Cell migration assay. Transfected cells ( $5 \times 10^{5}$ cells/well) were cultured in 6-well plates and were grown to $100 \%$ confluence. Cells were scratched with sterile $200 \mu \mathrm{l}$ pipette tips, and the plates were washed twice with Dulbecco's phosphate-buffered saline to remove the detached cells. Following this, the cells were incubated at $37^{\circ} \mathrm{C}$ in serum-free DMEM. During the following $24 \mathrm{~h}$, the cells migrated into the wounded area. Photographs were taken at 0 and $24 \mathrm{~h}$ using a light microscope (CKX41; Olympus Corporation, Tokyo, Japan).

Luciferase reporter gene assay. To clarify the target association between miR-34a and MALAT1, the MALAT1 sequences, containing the wild-type (WT) or mutated (MT) miR-34a binding sites, were subcloned into the pmiR-GLo luciferase reporter vector (Promega Corporation, Madison, WI, USA). T24 cells were co-transfected with WT or MT MALAT1 reporter plasmids, together with the miR-34a mimic or miR-NC, using Lipofectamine 2000. To clarify the target association between CCND1 and miR-34a, the CCND1 3'-UTR sequences, containing the WT or MT miR-34a binding sites, were also subcloned into the pmiR-GLo luciferase reporter vector. T24 cells were co-transfected with the WT or MT CCND1 reporter plasmids, together with the miR-34a mimic or miR-NC, using Lipofectamine 2000. At $48 \mathrm{~h}$ post-transfection, the luciferase activity was conducted using the Dual-Luciferase Reporter Assay System (Promega Corporation), according to the manufacturer's instructions. The ratio of Firefly luciferase activity to Renilla luciferase activity was determined.

Statistical analysis. Data were presented as the mean \pm standard deviation and were analysed using SPSS 19.0 software (IBM Corp., Armonk, NY, USA). A two-tailed Student's t-test was used to compare the difference between two groups. Multiple comparisons were performed using one-way analysis of variance followed by Tukey's post hoc test. Kaplan-Meier analysis with a log-rank test was performed to assess patient survival. The correlation between MALAT1
Table I. Association between MALAT1 expression and clinicopathological characteristics in bladder cancer.

\begin{tabular}{|c|c|c|c|c|}
\hline \multirow[b]{2}{*}{ Characteristics } & \multirow[b]{2}{*}{$\begin{array}{c}\text { Cases } \\
(n=56)\end{array}$} & \multicolumn{2}{|c|}{$\begin{array}{l}\text { MALAT1 } \\
\text { expression }\end{array}$} & \multirow[b]{2}{*}{ P-value } \\
\hline & & $\begin{array}{l}\text { High } \\
(n=29)\end{array}$ & $\begin{array}{c}\text { Low } \\
(n=27)\end{array}$ & \\
\hline Age & & & & 0.783 \\
\hline$<55$ & 21 & 10 & 11 & \\
\hline$\geq 55$ & 35 & 19 & 16 & \\
\hline Sex & & & & 0.790 \\
\hline Male & 34 & 17 & 17 & \\
\hline Female & 22 & 12 & 10 & \\
\hline $\begin{array}{l}\text { Lymph node } \\
\text { metastasis }\end{array}$ & & & & 0.013 \\
\hline Negative & 36 & 14 & 22 & \\
\hline Positive & 20 & 15 & 5 & \\
\hline TNM stage & & & & 0.007 \\
\hline I-II & 28 & 9 & 19 & \\
\hline III-IV & 28 & 20 & 8 & \\
\hline
\end{tabular}

TNM, Tumour, Node, Metastases; MALAT1, metastasis associated lung adenocarcinoma transcript 1.

and miR-34a expression was analysed using Spearman's rank correlation. $\mathrm{P}<0.05$ was considered to indicate a statistically significant difference.

\section{Results}

Upregulation of MALATl in BC tissues and cell lines. MALAT1 expression was first examined in 56 BC tissues and the matching adjacent non-tumour tissues using RT-qPCR. As indicated in Fig. 1A, the expression levels of MALAT1 were significantly increased in the BC tissues compared with the adjacent non-tumour tissues. Furthermore, the expression levels of MALAT1 were significantly increased in the BC tissues at stage III-IV compared with that in the tissues at stage I-II (Fig. 1B). These patients with BC were subsequently divided into high- and low-MALAT1 expression groups based on the mean expression value in the BC tissues. As indicated in Table I, high expression of MALAT1 was significantly associated with advanced Tumour, Node, Metastases (TNM) stage and positive lymph node metastasis. Furthermore, patients with high MALAT1 expression exhibited a shorter survival time when compared with those with a low MALAT1 expression (Fig. 1C). The expression of MALAT1 in the BC cell lines (HT-1376, RT112, 253J and T24) and the normal urinary tract epithelial cell line SV-HUC-1 were also assessed. MALAT1 was also significantly upregulated in the BC cell lines compared with the SV-HUC-1 cells (Fig. 1D).

Inhibition of MALAT1 reduces the proliferation and migration of $B C$ cells. The function of MALAT1 in BC was studied using T24 cells. MALAT1-specific siRNA was 
A

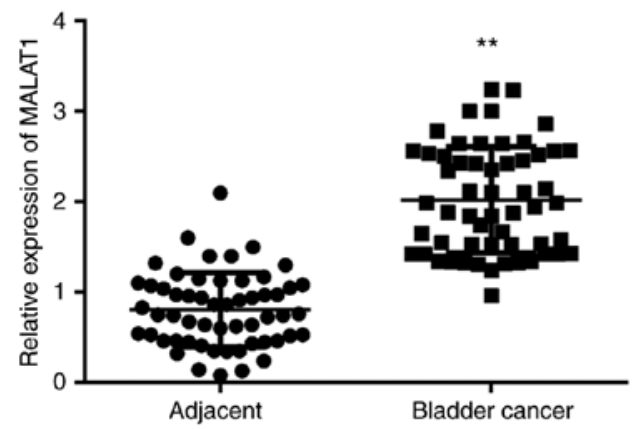

C

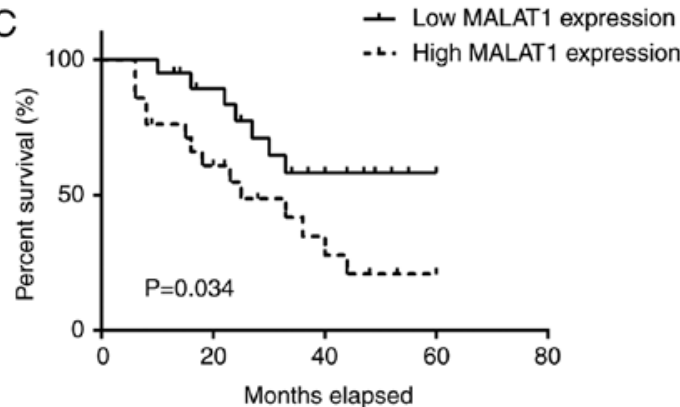

B
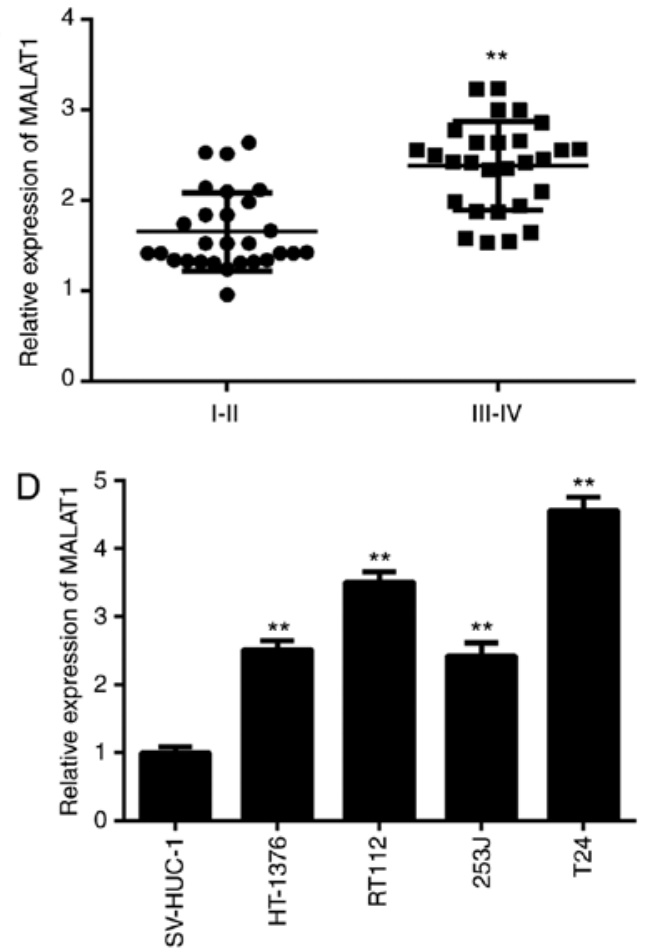

Figure 1. Upregulation of MALAT1 in BC tissues and cell lines. (A) RT-qPCR data indicated that MALAT1 was upregulated in the BC tissues when compared with the adjacent non-tumour tissues. ${ }^{* *} \mathrm{P}<0.01$ vs. adjacent. (B) MALAT1 was upregulated in the BC tissues at stage III-IV when compared with that in the tissues at stage I-II. ${ }^{* *} \mathrm{P}<0.01$ vs. I-II. (C) Patients with BC exhibiting high MALAT1 expression had a shorter survival time when compared with those with low MALAT1 expression. (D) RT-PCR data indicated that MALAT1 was significantly upregulated in the BC cell lines compared with the SV-HUC-1 cells. ${ }^{* *} \mathrm{P}<0.01$ vs. SV-HUC-1. BC, bladder cancer; MALAT1, metastasis associated lung adenocarcinoma transcript 1; RT-qPCR, reverse transcription-quantitative polymerase chain reaction.

A

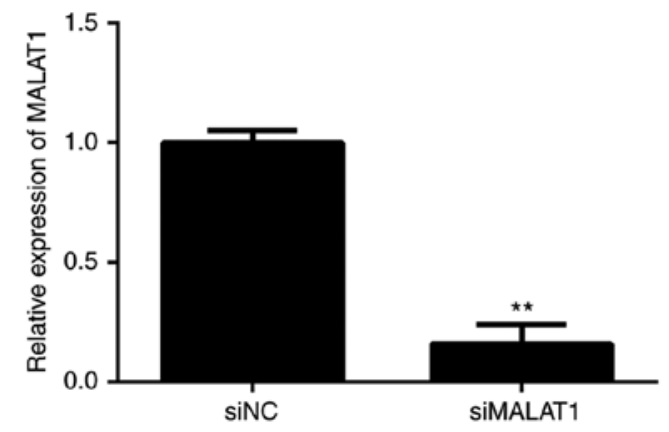

C

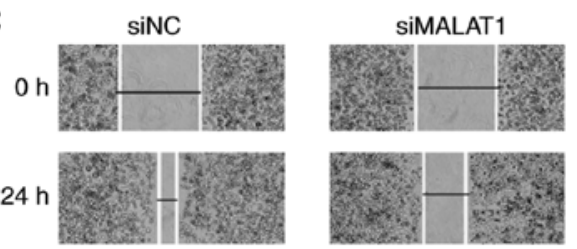

B
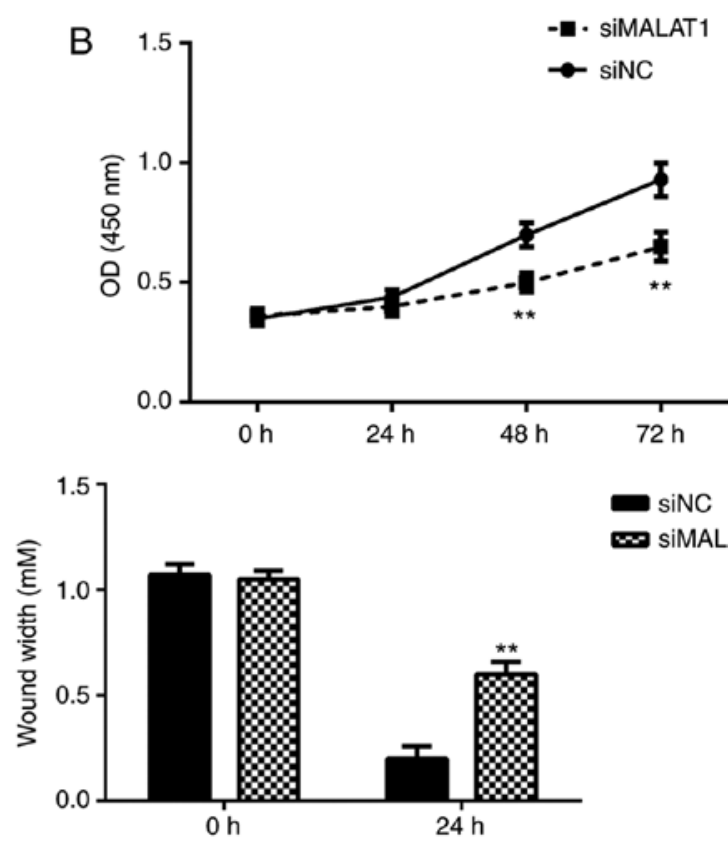

Figure 2. Inhibition of MALAT1 reduces the proliferation and migration of bladder cancer cells. T24 cells were transfected with a NC siRNA or MALAT1 siRNA. Following transfection, (A) the expression of MALAT1 was assessed using polymerase chain reaction. (B) The Cell Counting Kit-8 assay and (C) wound healing assay were used to study cell proliferation and migration, respectively (magnification, $\mathrm{x} 40$ ). ${ }^{* *} \mathrm{P}<0.01$ vs. siNC. MALAT1, metastasis associated lung adenocarcinoma transcript 1; OD, optical density; NC, negative control; si, small interfering RNA.

transfected into T24 cells to inhibit its expression. As indicated in Fig. 2A, MALAT1 was significantly downregulated in the siMALAT1 group compared with the siNegative
Control (siNC) group. These data confirmed that the transfection efficiency was successful. Data from CKK-8 and wound healing assays further indicated that the knockdown 


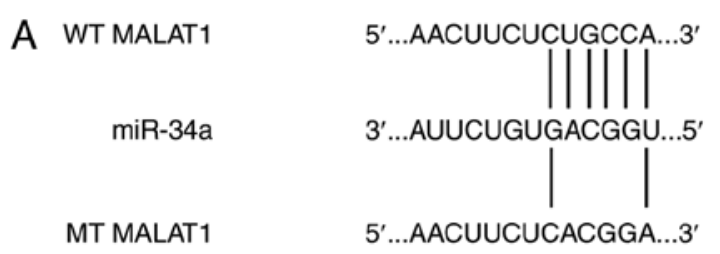

MT MALAT1
MALAT1-WT
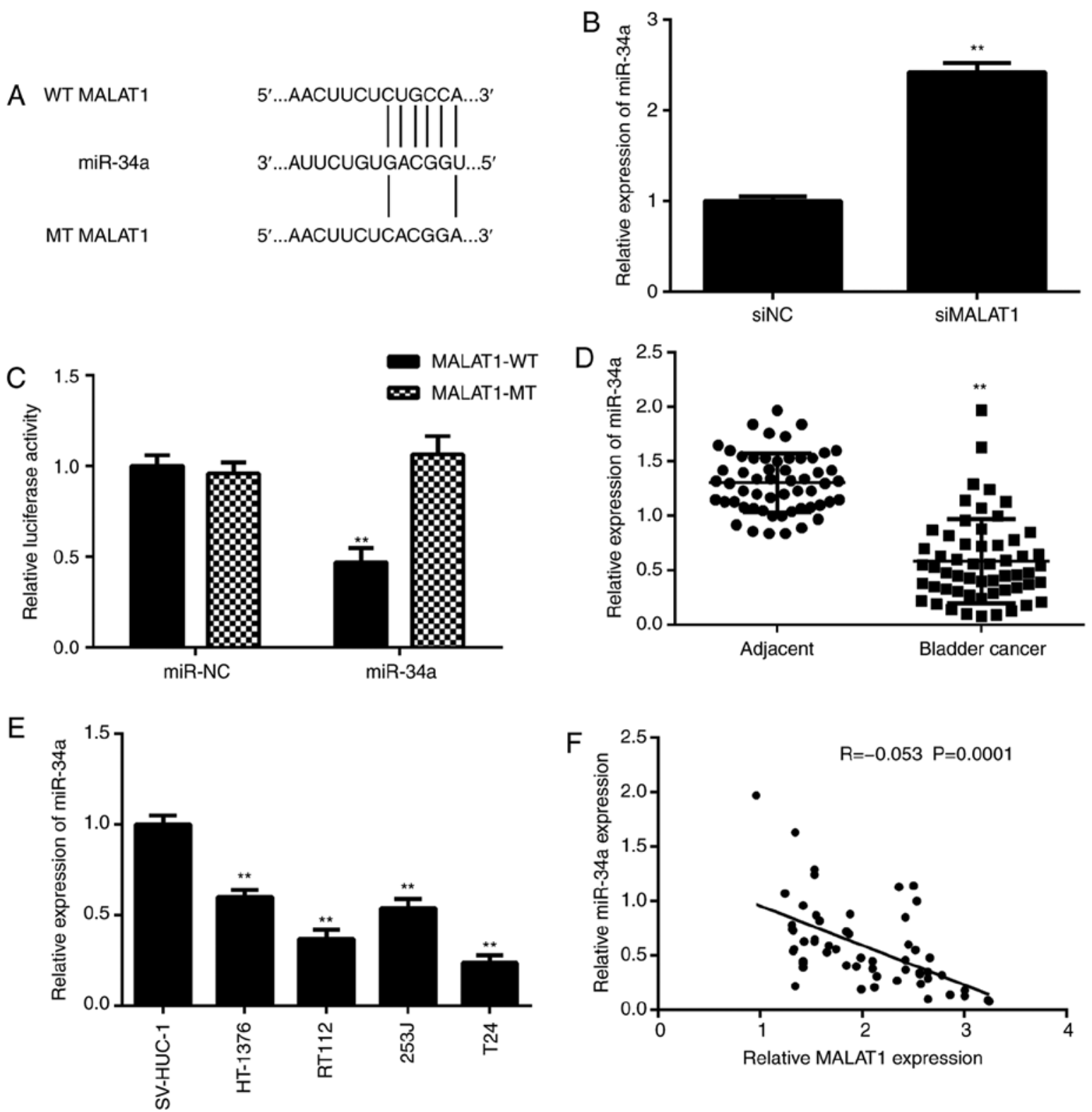

Figure 3. miR-34a is a target of MALAT1 in bladder cancer cells. (A) miR-34a had a potential binding site in MALAT1. (B) Knockdown of MALAT1 increased the expression of miR-34a in the T24 cells. ${ }^{* *} \mathrm{P}<0.01 \mathrm{vs}$. siNC. (C) The transfection with the miR-34a mimics significantly decreased the luciferase activity of the MALAT1-WT luciferase reporter gene plasmid but had no effect on the luciferase activity of the MALAT1-MT luciferase reporter gene plasmid in the T24 cells. " $\mathrm{P}<0.01$ vs. miR-NC. (D) RT-qPCR data revealed that miR-34a was downregulated in the BC tissues compared with the adjacent non-tumour tissues. ${ }^{* *} \mathrm{P}<0.01$ vs. adjacent. (E) RT-qPCR data also indicated that miR-34a was downregulated in the BC cell lines when compared with the SV-HUC-1 cells. ${ }^{* *} \mathrm{P}<0.01$ vs. SV-HUC-1. (F) An inverse correlation between the expression of miR-34a and MALAT1 in the BC tissues. MALAT1, metastasis associated lung adenocarcinoma transcript 1; miR, microRNA; WT, wild-type; MUT, mutant; NC, negative control; si, small interfering RNA; RT-qPCR, reverse transcription-quantitative polymerase chain reaction.

of MALAT1 significantly suppressed the proliferation and migration of T24 cells (Fig. 2B and C).

miR-34a is a target of MALAT1 in BC cells. A bioinformatic analysis was conducted to predict the potential MALAT1-miR interactions. As indicated in Fig. 3A, miR-34a had a potential binding site in MALAT1. Consistent with this prediction, the inhibition of MALAT1 expression significantly increased the expression of miR-34a in T24 cells (Fig. 3B). To further confirm this prediction, a luciferase reporter gene assay was performed using the T24 cells, and the data revealed that transfection with the miR-34a mimics significantly decreased the luciferase activity of the MALAT1-WT luciferase reporter gene plasmid, but had no significant effect on the luciferase activity of the MALAT1-MT luciferase reporter gene plasmid in the T24 cells (Fig. 3C). These findings demonstrated that miR-34a and MALAT1 were associated. Following this, it was indicated that the expression levels of miR-34a were significantly reduced in the $\mathrm{BC}$ tissues and cell lines compared with the adjacent non-tumour tissues and SV-HUC-1 cells, respectively (Fig. 3D and E). Furthermore, an inverse correlation between the expression of miR-34a and MALAT1 in the BC tissues was observed (Fig. 3F).

miR-34a is involved in MALAT1-mediated BC cell proliferation and migration. It was speculated that miR-34a may be involved 

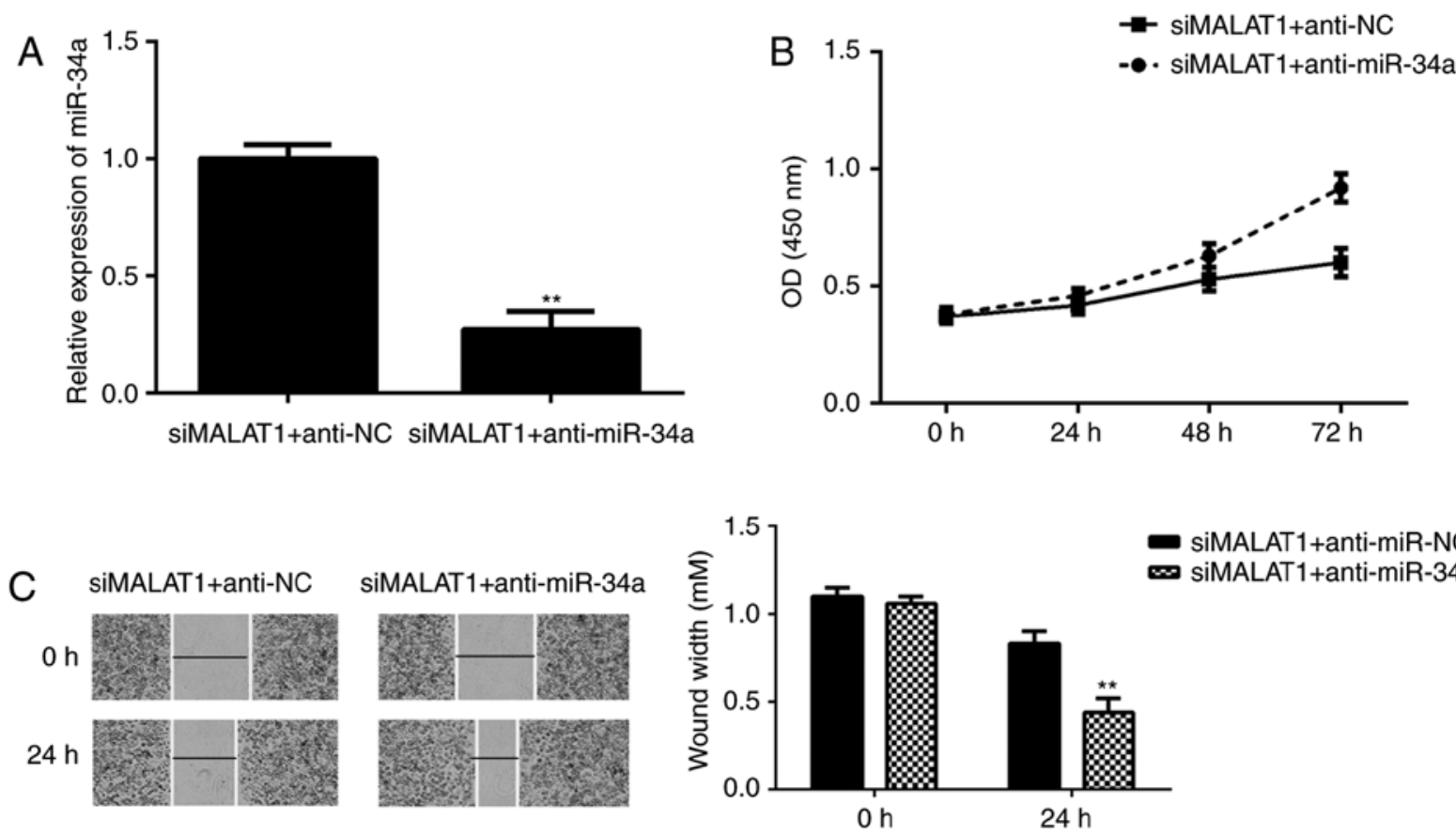

Figure 4. miR-34a is involved in MALAT1-mediated bladder cancer cell proliferation and migration. The siMALAT1-transfected T24 cells were transfected with the miR-34a inhibitor or NC inhibitor. Following transfection, (A) polymerase chain reaction was used to examine the expression of MALAT1. (B) The Cell Countin Kit 8 assay and (C) wound healing assay were used to study cell proliferation and migration, respectively (magnification, $x 40){ }^{* *} \mathrm{P}<0.01 \mathrm{vs}$. siMALAT1+anti-NC. miR, microRNA; MALAT1, metastasis associated lung adenocarcinoma transcript 1; NC, negative control.

in MALAT1-mediated BC cell proliferation and migration. To clarify this speculation, siMALAT1-transfected T24 cells were transfected with miR-34a inhibitor or NC inhibitor. The qPCR data indicated that miR-34a was significantly downregulated in the siMALAT1+anti-miR-34a group compared with the siMALAT1+anti-NC group (Fig. 4A). These data confirmed that the transfection efficiency was successful. Further study revealed that the proliferation and migration of T24 cells were significantly increased in the siMALAT1+anti-miR-34a group compared with the siMALAT1+anti-NC group (Fig. 4B and C), which suggested that knockdown of miR-34a impaired the suppressive effects on the T24 cell proliferation and migration induced by MALAT1 downregulation.

CCND1 is a target gene of miR-34a in BC cells. Bioinformatic analysis data demonstrated that CCND1 was a target gene of miR-34a (Fig. 5A). The luciferase reporter gene assay was conducted to confirm this prediction, and the data indicated that transfection with the miR-34a mimics significantly downregulated the luciferase activity of the CCND1-WT luciferase reporter gene plasmid, but had no significant effect on the luciferase activity of the CCND1-MT luciferase reporter gene plasmid (Fig. 5B). These findings demonstrated that miR-34a was a target gene of miR-34a. In addition, it was revealed that the inhibition of MALAT1 expression caused a reduction in CCND1 expression in T24 cells, which was abolished by the knockdown of miR-34a (Fig. 5C and D). These findings suggested that MALAT1 may positively regulate the expression of CCND1 via directly targeting miR-34a in T24 cells. Following this, it was investigated whether CCND1 was involved in miR-34a-mediated BC growth. T24 cells were co-transfected with miR-NC and blank vector (miR-NC+blank), miR-34a mimics and blank vector (miR-34a+blank), and miR-34a mimics and CCND1 plasmid (miR-34a+CCND1). CKK-8 assay data demonstrated that the proliferation of T24 cells was significantly reduced in the miR-34a+blank group when compared with the miR-NC+blank group; however, proliferation was restored in the miR-34a+CCND1 group (Fig. 5E). These findings suggested that miR-34a inhibits BC cell proliferation, and upregulation of CCND1 impaired the suppressive effects of miR-34a on T24 cell proliferation.

CCND1 acts as a downstream effector of MALAT1 in BC cells. Based on the above findings, it was speculated that CCND1 may be a downstream effector of MALAT1 in BC cells. To clarify this speculation, siMALAT1-transfected T24 cells were transfected with a CCND1 expression plasmid or a blank vector. As indicated in Fig. 6A and B, CCND1 mRNA and protein expression levels were significantly upregulated in the siMALAT1+CCND1 group compared with the siMALAT1+blank group. These data confirmed that the transfection efficiency was successful. Further investigation identified that cell proliferation and migration were also significantly increased in the siMALAT1+CCND1 group compared with the siMALAT1+blank (Fig. 6C and D), which suggested that CCND1 upregulation may impair the inhibitory effects of MALAT1 inhibition on T24 cell proliferation and migration. Therefore, the results suggested that CCND1 acted as a downstream effector of MALAT1 in BC cells.

CCND1 is upregulated in BC. Finally, it was observed that the expression of CCND1 was significantly increased in the $\mathrm{BC}$ tissues and cell lines compared with the adjacent 

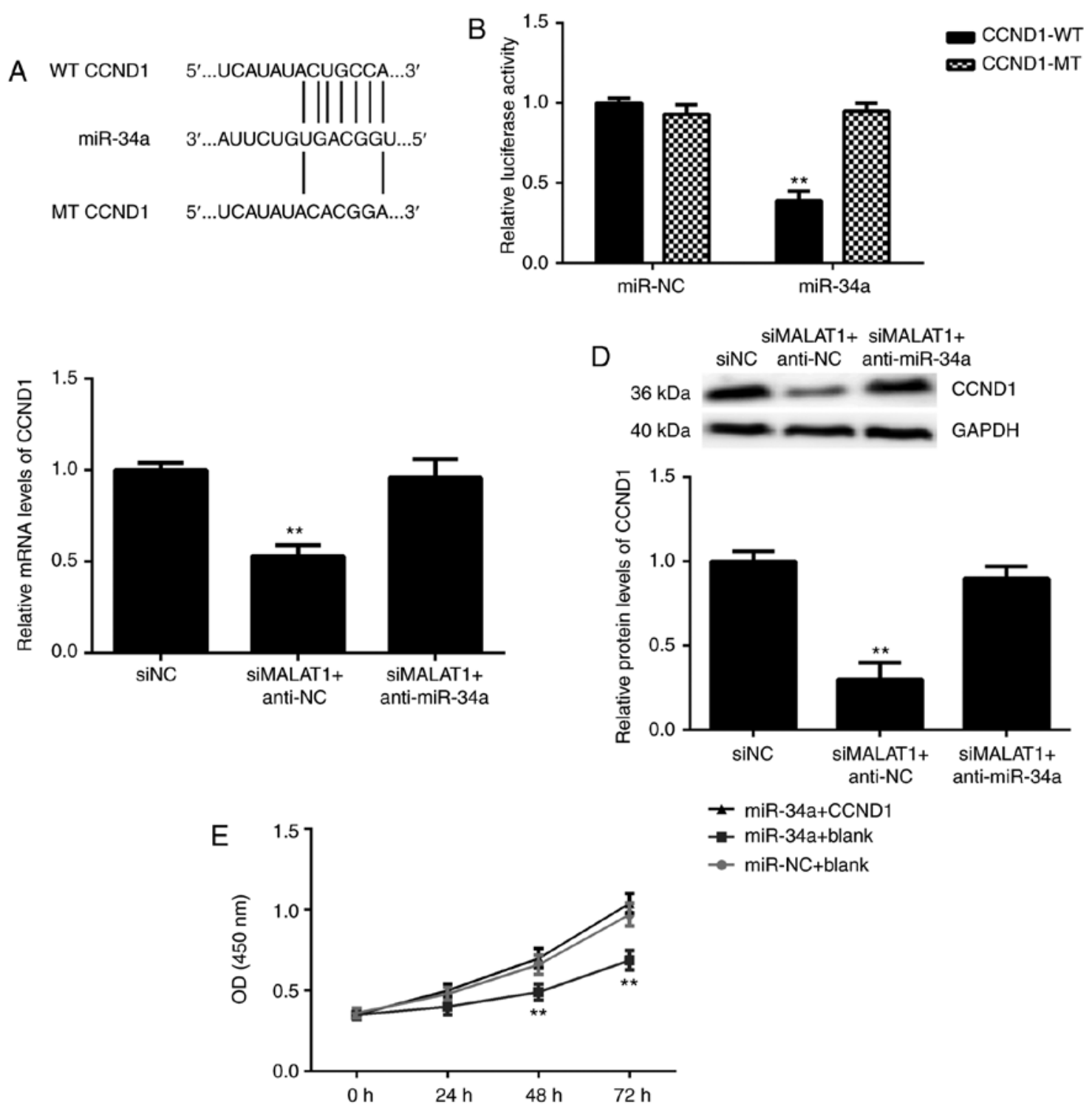

Figure 5. CCND1 is a target gene of miR-34a in bladder cancer cells. (A) miR-34a had a potential binding site in CCND1. (B) Transfection with the miR-34a mimics significantly downregulated the luciferase activity of the CCND1-WT luciferase reporter gene plasmid, but had no significant effect on the luciferase activity of the CCND1-MT luciferase reporter gene plasmid. ${ }^{* *} \mathrm{P}<0.01$ vs. miR-NC. (C and D) Knockdown of MALAT1 caused a reduction in the mRNA and protein expression levels of CCND1 in T24 cells, which was abolished by the knockdown of miR-34a. ${ }^{* *} \mathrm{P}<0.01$ vs. siNC. (E) T24 cells were co-transfected with miR-NC and blank vector (miR-NC+blank), miR-34a mimics and blank vector (miR-34a+blank), and miR-34a mimics and CCND1 plasmid (miR-34a+CCND1). The Cell counting Kit-8 assay was then conducted to study cell proliferation. ${ }^{* *} \mathrm{P}<0.01$ vs. miR-NC+blank. MALAT1, metastasis associated lung adenocarcinoma transcript 1; miR, microRNA; WT, wild-type; MUT, mutant; NC, negative control; si, small interfering RNA; CCND1, cyclin D1.

non-tumour tissues and the normal urinary tract epithelial cell line SV-HUC-1 (Fig. 7). Therefore, the findings suggested that CCND1 was upregulated in BC.

\section{Discussion}

The regulatory mechanism of MALAT1 underlying BC growth and metastasis has not been fully elucidated. The present study indicated that MALAT1 was significantly upregulated in the $\mathrm{BC}$ tissues and cell lines compared with that in adjacent non-tumour tissues and the normal urinary tract epithelial cell line SV-HUC-1. Furthermore, the expression levels of MALAT1 were significantly higher in the BC tissues at stage III-IV when compared with the tissues at stage I-II. Notably, the knockdown of MALAT1 significantly inhibited
$\mathrm{BC}$ cell proliferation and migration by targeting miR-34a. In addition, the expression of miR-34a was significantly decreased in the BC tissues and cell lines compared with the adjacent non-tumour tissues and the SV-HUC-1 cells. Furthermore, the expression of miR-34a was inversely correlated with the expression of MALAT1 in the BC tissues. CCND1 was then identified as a target gene of miR-34a, and its expression was negatively mediated by miR-34a in the BC cells. Notably, the upregulation of CCND1 impaired the effect of MALAT1 inhibition on $\mathrm{BC}$ cell proliferation and migration. In addition, the expression of CCND1 was significantly increased in the $\mathrm{BC}$ tissues and cell lines.

An increasing number of lncRNAs have been demonstrated to function as key regulators in various types of human cancer, including BC. For instance, lncRNA activated by TGF- $\beta$ 

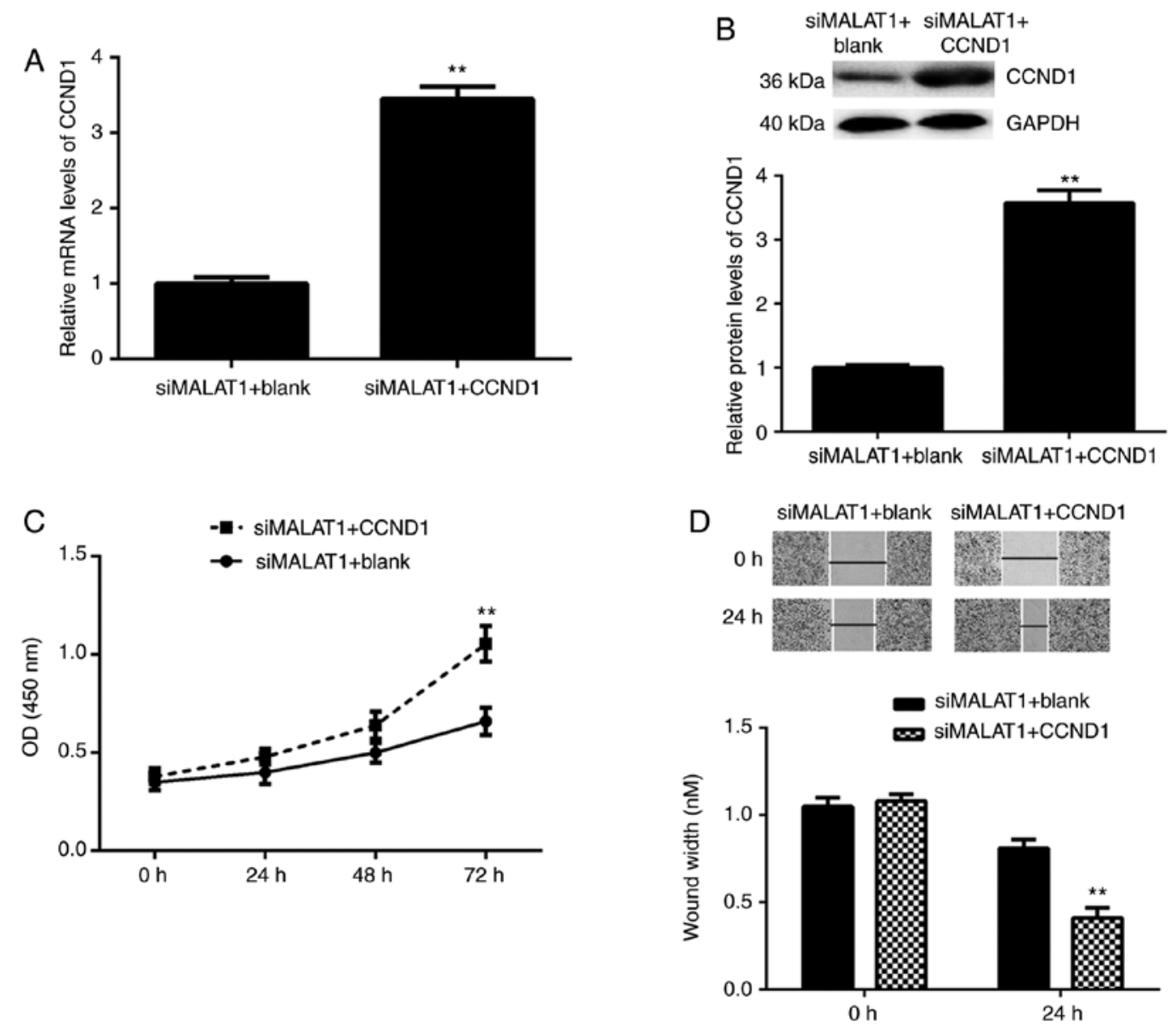

Figure 6. CCND1 acts as a downstream effector of MALAT1 in bladder cancer cells. siMALAT1-transfected T24 cells were transfected with the CCND1 expression plasmid or a blank vector. Following transfection, (A) reverse transcription-quantitative polymerase chain reaction and (B) western blot analysis assays were used to examine the mRNA and protein expression levels of CCND1, respectively. (C) The Cell Counting Kit-8 assay and (D) wound healing assay were used to study cell proliferation and migration, respectively (magnification, $\mathrm{x} 40$ ). ${ }^{* *} \mathrm{P}<0.01$ vs. siMALAT1+blank. MALAT1, metastasis associated lung adenocarcinoma transcript 1; miR, microRNA; si, small interfering RNA; CCND1, cyclin D1.

\section{A}

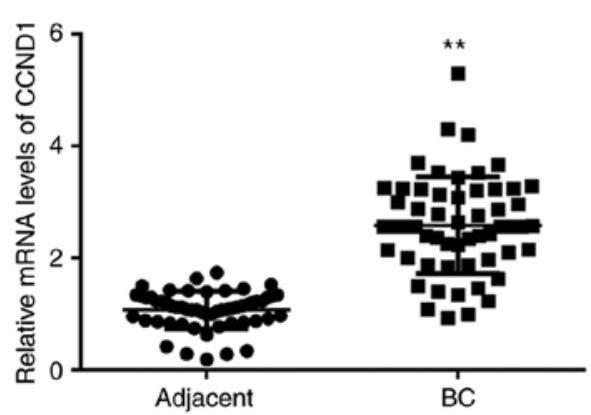

C

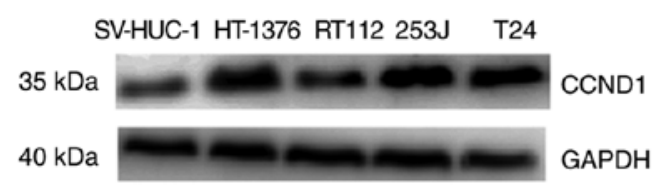

B
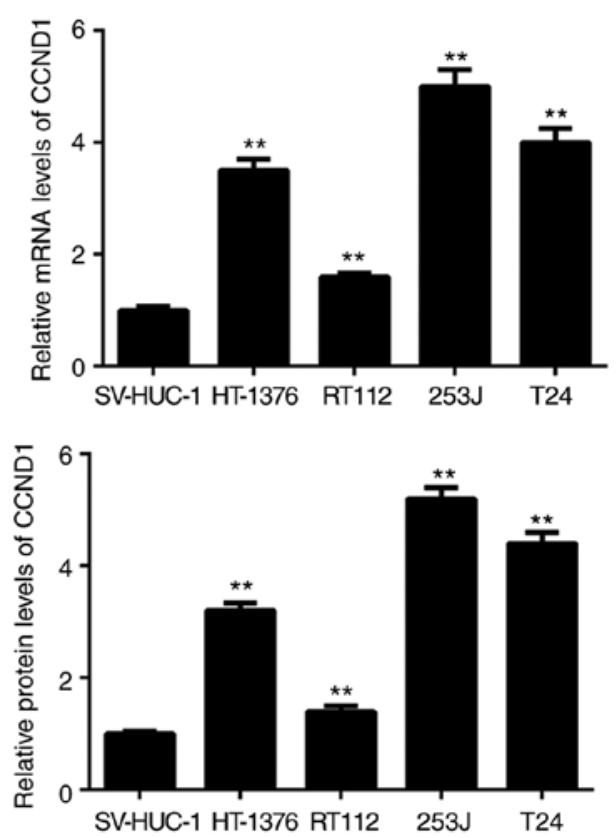

Figure 7. CCND1 is upregulated in BC. (A) RT-qPCR data indicated that the mRNA expression of CCND1 was increased in the BC tissues compared with the adjacent non-tumour tissues. ${ }^{* *} \mathrm{P}<0.01$ vs. adjacent. (B) RT-qPCR and (C) western blot analysis data indicated that the mRNA and protein expression levels of CCND1, respectively, were increased in the BC cell lines compared with the SV-HUC-1 cells ${ }^{* *} \mathrm{P}<0.01$ vs. SV-HUC-1. BC, bladder cancer; RT-qPCR, reverse transcription-quantitative polymerase chain reaction; CCND1, cyclin D1. 
promotes $\mathrm{BC}$ cell proliferation, migration and invasion by suppressing the expression of miR-126 (8). LncRNA growth arrest-specific 5 promotes BC cell apoptosis by inhibiting the transcription of Ezh2 (27). The findings in the present study revealed that MALAT1 was significantly upregulated in BC tissues and cell lines, and its upregulation was significantly associated with advanced TNM stage, lymph node metastasis and a shorter survival time in patients with BC. Consistent with these data, Li et al (16) also reported that the high expression of MALAT1 promoted BC progression and metastasis and was associated with poor prognosis. In the present study, it was revealed that the knockdown of MALAT1 decreased BC cell proliferation and migration. Similarly, Jiao et al (28) reported that the overexpression of MALAT1 promoted the proliferation, migration and invasion of bladder transitional cell carcinoma cells.

LncRNAs typically function by regulating miR expression and, thus, affect downstream target genes $(12,29)$. For instance, the lncRNA X-inactive specific transcript promotes TGF- $\beta$-induced EMT in NSCLC cells by regulating the miR-367/141-zinc finger E-box binding homeobox 2 axis (30). LncRNA taurine up-regulated 1 promotes oral squamous cell carcinoma progression by upregulating formin like 2 by sequestering miR-219 (31). In the present study, knockdown of MALAT1 increased miR-34a expression in T24 cells, and it was confirmed that MALAT1 directly targeted miR-34a by using a luciferase reporter gene assay. Furthermore, miR-34a was downregulated in BC, and its downregulation was inversely correlated with the increased expression of MALAT1 in the BC tissues. These findings suggested that the decreased expression of miR-34a may be due to the increased expression of MALAT1 in BC. The present findings suggested that the suppressive effects of MALAT1 downregulation on the proliferation and migration of the T24 cells was through the upregulation of miR-34a.

Bioinformatic analysis was performed to study the potential target gene of miR-34a in BC cells, and CCND1 was selected. The result of the luciferase reporter gene assay indicated that CCND1 was a direct target gene of miR-34a in T24 cells. Notably, several previous studies also confirmed targeting associations in other cancer types. For instance, Ye et al (32) indicated that the inhibition of miR-34a promoted the proliferation and migration of laryngeal carcinoma cells by targeting CCND1. In addition, miR-34a overexpression induced significant G1 cell-cycle arrest in NSCLC cells via the inhibition of CCND1 (33). In the present study, the inhibition of MALAT1 downregulated the expression of CCND1 in the T24 cells, which was impaired by the silencing of miR-34a, suggesting that MALAT1 impacted CCND1 expression via miR-34a. Furthermore, CCND1 overexpression eliminated the inhibitory effects on T24 cell proliferation and migration induced by MALAT1 downregulation, suggesting that CCND1 was a downstream effector of MALAT1 in BC cells.

To the best of our knowledge, the present study indicated for the first time that MALAT1, which is upregulated in BC, promotes tumour cell proliferation and migration via modulating the miR-34a/CCND1 axis. Thus, the findings suggest that MALAT1 may be a potential therapeutic target for BC treatment.

\section{Acknowledgements}

Not applicable.

\section{Funding}

No funding was received.

\section{Availability of data and materials}

All data generated or analyzed during this study are included in this published article.

\section{Authors' contributions}

YL collected clinical tissues and wrote the manuscript. YL, SG and QD performed the experiments. QZ designed the study and revised the manuscript.

\section{Ethics approval and consent to participate}

The present study was been approved by the Ethics Committee of First People's Hospital of Jining City (Jining, China). All patients provided written informed consent.

\section{Patient consent for publication}

All patients provided written informed consent.

\section{Competing interests}

The authors declare that they have no competing interests.

\section{References}

1. Siegel RL, Miller KD and Jemal A: Cancer statistics, 2015. CA Cancer J Clin 65: 5-29, 2015.

2. Skeldon SC and Larry Goldenberg S: Bladder cancer: A portal into mens health. Urol Oncol 33: 40-44, 2015

3. Sathe A and Nawroth R: Targeting the PI3K/AKT/mTOR pathway in bladder cancer. Methods Mol Biol 1655: 335-350, 2018.

4. Zhou Y, Meng X, Chen S, Li W, Li D, Singer R and Gu W: IMP1 regulates UCA1-mediated cell invasion through facilitating UCA1 decay and decreasing the sponge effect of UCA1 for miR-122-5p. Breast Cancer Res 20: 32, 2018.

5. Smolle MA and Pichler M: The role of long non-coding RNAs in osteosarcoma. Noncoding RNA 4: E7, 2018.

6. Ruan X: Long noncoding RNA central of glucose homeostasis. J Cell Biochem 117: 1061-1065, 2016.

7. Wapinski $\mathrm{O}$ and Chang HY: Long noncoding RNAs and human disease. Trends Cell Biol 21: 354-361, 2011.

8. Zhai $\mathrm{X}$ and $\mathrm{Xu} \mathrm{W}$ : Long noncoding RNA ATB promotes proliferation, migration and invasion in bladder cancer by suppressing microRNA-126. Oncol Res 26: 1063-1072, 2018.

9. Xu R, Zhu X, Chen F, Huang C, Ai K, Wu H, Zhang L and Zhao X: LncRNA XIST/miR-200c regulates the stemness properties and tumourigenicity of human bladder cancer stem cell-like cells. Cancer Cell Int 18: 41, 2018.

10. Peng Z, Liu C and Wu M: New insights into long noncoding RNAs and their roles in glioma. Mol Cancer 17: 61, 2018.

11. Zhang Y, Dai Q, Zeng F and Liu H: MALAT1 Promotes the proliferation and metastasis of osteosarcoma cells by activating the Rac1/JNK pathway via targeting miR-509. Oncol Res: Apr 27, 2018 (Epub ahead of print). doi: 10.3727/096504017X1 4957939026111.

12. Liu S, Song L, Zeng S and Zhang L: MALAT1-miR-124-RBG2 axis is involved in growth and invasion of HR-HPV-positive cervical cancer cells. Tumour Biol 37: 633-640, 2016. 
13. Ji Q, Liu X, Fu X, Zhang L, Sui H, Zhou L, Sun J, Cai J, Qin J, Ren J and Li Q: Resveratrol inhibits invasion and metastasis of colorectal cancer cells via MALAT1 mediated Wnt/beta-catenin signal pathway. PLoS One 8: e78700, 2013.

14. Hirata H, Hinoda Y, Shahryari V, Deng G, Nakajima K, Tabatabai ZL, Ishii N and Dahiya R: Long noncoding RNA MALAT1 promotes aggressive renal cell carcinoma through Ezh2 and interacts with miR-205. Cancer Res 75: 1322-1331, 2015.

15. Ying L, Chen Q, Wang Y, Zhou Z, Huang Y and Qiu F: Upregulated MALAT-1 contributes to bladder cancer cell migration by inducing epithelial-to-mesenchymal transition. Mol Biosyst 8: 2289-2294, 2012

16. Li C, Cui Y, Liu LF, Ren WB, Li QQ, Zhou X, Li YL, Li Y, Bai XY and Zu XB: High expression of long noncoding RNA MALAT1 indicates a poor prognosis and promotes clinical progression and metastasis in bladder cancer. Clin Genitourin Cancer 15: 570-576, 2017.

17. Xie H, Liao X, Chen Z, Fang Y, He A, Zhong Y, Gao Q, Xiao H, Li J, Huang W and Liu Y: LncRNA MALAT1 inhibits apoptosis and promotes invasion by antagonizing miR-125b in bladder cancer cells. J Cancer 8: 3803-3811, 2017.

18. Farazi TA, Hoell JI, Morozov P and Tuschl T: MicroRNAs in human cancer. Adv Exp Med Biol 774: 1-20, 2013.

19. Garg D and Cohen SM: miRNAs and aging: A genetic perspective. Ageing Res Rev 17: 3-8, 2014.

20. Boon RA and Vickers KC: Intercellular transport of microRNAs. Arterioscler Thromb Vasc Biol 33: 186-192, 2013.

21. Hwang HW and Mendell JT: MicroRNAs in cell proliferation, cell death, and tumorigenesis. Br J Cancer 94: 776-780, 2006.

22. Ma ZL, Hou PP, Li YL, Wang DT, Yuan TW, Wei JL, Zhao BT, Lou JT, Zhao XT, Jin Y and Jin YX: MicroRNA-34a inhibits the proliferation and promotes the apoptosis of non-small cell lung cancer H1299 cell line by targeting TGFbetaR2. Tumour Biol 36 : 2481-2490, 2015.

23. Wu X,Zhong D, Gao Q, Zhai W, Ding Z and Wu J: MicroRNA-34a inhibits human osteosarcoma proliferation by downregulating ether a go-go 1 expression. Int J Med Sci 10: 676-682, 2013.

24. Yu G, Yao W, Xiao W, Li H, Xu H and Lang B: MicroRNA-34a functions as an anti-metastatic microRNA and suppresses angiogenesis in bladder cancer by directly targeting CD44. J Exp Clin Cancer Res 33: 779, 2014.
25. Sun H, Tian J, Xian W, Xie T and Yang X: miR-34a inhibits proliferation and invasion of bladder cancer cells by targeting orphan nuclear receptor HNF4G. Dis Markers 2015: 879254, 2015.

26. Livak KJ and Schmittgen TD: Analysis of relative gene expression data using real-time quantitative PCR and the 2(-Delta Delta C(T)) method. Methods 25: 402-408, 2001

27. Wang M, Guo C, Wang L, Luo G, Huang C, Li Y, Liu D, Zeng F, Jiang $\mathrm{G}$ and Xiao $\mathrm{X}$ : Long noncoding RNA GAS5 promotes bladder cancer cells apoptosis through inhibiting EZH2 transcription. Cell Death Dis 9: 238, 2018.

28. Jiao D, Li Z, Zhu M, Wang Y, Wu G and Han X: LncRNA MALAT1 promotes tumor growth and metastasis by targeting miR-124/foxq1 in bladder transitional cell carcinoma (BTCC). Am J Cancer Res 8: 748-760, 2018.

29. Xie CH, Cao YM, Huang Y, Shi QW, Guo JH, Fan ZW, Li JG, Chen BW and Wu BY: Long non-coding RNA TUG1 contributes to tumorigenesis of human osteosarcoma by sponging miR-9-5p and regulating POU2F1 expression. Tumour Biol 37: 15031-15041, 2016.

30. Li C, Wan L, Liu Z, Xu G, Wang S, Su Z, Zhang Y, Zhang C, Liu X, Lei Z and Zhang HT: Long non-coding RNA XIST promotes TGF-beta-induced epithelial-mesenchymal transition by regulating miR-367/141-ZEB2 axis in non-small-cell lung cancer. Cancer Lett 418: 185-195, 2018.

31. Yan G, Wang X, Yang M, Lu L and Zhou Q: Long non-coding RNA TUG1 promotes progression of oral squamous cell carcinoma through upregulating FMNL2 by sponging miR-219. Am J Cancer Res 7: 1899-1912, 2017.

32. Ye J, Li L, Feng P, Wan J and Li J: Downregulation of miR-34a contributes to the proliferation and migration of laryngeal carcinoma cells by targeting cyclin D1. Oncol Rep 36: 390-398, 2016.

33. Sun F, Fu H, Liu Q, Tie Y, Zhu J, Xing R, Sun Z and Zheng X: Downregulation of CCND1 and CDK6 by miR-34a induces cell cycle arrest. FEBS Lett 582: 1564-1568, 2008. 